\title{
Crecimiento económico y ecoinnovación en la Unión Europea
}

\author{
Edgar J. Saucedo \\ Enrique Hernández \\ Universidad Veracruzana \\ Samantha Rullán \\ Cornell University
}

\section{Resumen}

El objetivo del texto es mostrar la relación entre ecoinnovación y crecimiento económico para los países de la Unión Europea. Para ello, analizamos estudios teóricos sobre innovación (y ecoinnovación) y el crecimiento económico, para posteriormente hacer una revisión de las políticas de la Unión Europea en relación a la ecoinnovación. Nuestros resultados muestran que la ecoinnovación tiene un impacto positivo sobre el crecimiento económico en la Unión Europea en el periodo 2002-2010, debido a que el gasto en inversión en el medio ambiente de los gobiernos, los ingresos fiscales ambientales y las exportaciones de productos de ecoindustrias tienen un impacto positivo con el crecimiento económico. Nuestra conclusión es que las políticas públicas que incentivan la ecoinnovación podrían tener un impacto positivo en el crecimiento económico.

Palabras clave: ecoinnovación, crecimiento económico, Unión Europea.

Clasificación JEL: O44, O47, O52.

\begin{abstract}
The aim of the paper is to show the relationship between ecoinnovation and economic growth for the countries of the European Union. For that, we analyze theoretical studies on innovation (and ecoinnovation) and economic growth, later we review the European Union ecoinnovation policies. Our results show that ecoinnovation has a positive impact on economic growth in the European Union in the period 2002-2010, due to investment in the environment (of governments), environmental tax revenues and ecoproducts exports have a positive impact on economic growth. Our conclusion is that public policies that encourage ecoinnovation could have a positive impact on economic growth.
\end{abstract}

Keywords: ecoinnovation, economic growth, European Union.

JEL classification: $044,047,052$.

\section{Introducción}

La alta degradación ambiental que ha aumentado en las últimas décadas, ha provocado la necesidad de que los gobiernos nacionales introduzcan políticas públicas que reduzcan dicha tendencia, con el objetivo de que en el mediano y largo plazo se cuente con un desarrollo sostenible. Por otro lado, a nivel internacional se han 
llevado a cabo varias conferencias con el objetivo de frenar una tendencia creciente de daño a los ecosistemas, sobre todo con lo relacionado con el cambio climático, debido a que ha sido considerado como una externalidad global, por lo que se ha hecho de suma importancia las estrategias de cooperación internacional.

Las políticas públicas que tienen como objetivo una mejora del medio ambiente producen costos en términos económicos, que van desde los gastos de las empresas hasta el gasto público de las política públicas ambientales. Dichos costos pueden ser considerablemente altos, lo que puede generar incentivos a no realizar actividades en pro del medio ambiente por parte de las empresas y los gobiernos.

La ecoinnovación permite reducir los costos de una mejora al medio ambiente, debido a que se produce una innovación y un menor impacto a los ecosistemas al mismo tiempo, por lo que se obtienen ingresos extras derivados de la innovación (que tienen que ver con el poder monopólico que tiene la empresa en lo que dura la patente), que podrían cubrir una parte considerable del costo para mejorar el medio ambiente.

La Unión Europea es una de las regiones del mundo que mayor importancia le da al cuidado al medio ambiente en términos de la cooperación entre sus Estados miembros y las políticas supranacionales que llevan a cabo las instituciones europeas. En este sentido, la Unión Europea ha lanzado el Plan de Acción de la Ecoinnovación en 2011, que tiene como objetivo la promoción de la innovación en temas ambientales, además de que la Estrategia Europea 2020 tiene busca un crecimiento económico inteligente, sostenible e integrador.

En términos empíricos, hay varios estudios que relacionan el crecimiento económico y la innovación (Ulku, 2004; Higino, 2005; Hasan y Tucci, 2010); sin embargo, en términos de ecoinnovación se han enfocado más a las empresas (Kesidou y Demirel, 2011; Kesidou y Demirel, 2012), por lo que es necesario relacionar el crecimiento económico y la ecoinnovación a nivel de países.

En este trabajo analizamos si la ecoinnovación tiene relación con el crecimiento económico entre los Estados miembros de la Unión Europea para el periodo 20022010. La metodología utilizada es una regresión con datos panel con efectos aleatorios para 11 países de la Unión Europea, debido a que no fue posible conseguir un panel completo para todos los países en dicho periodo de tiempo. El objetivo del texto es analizar empíricamente si la ecoinnovación tiene un impacto positivo sobre el crecimiento económico de 11 países de la Unión Europea.

Después de la introducción, en la siguiente sección se señalan los aspectos teóricos de la relación entre innovación (y ecoinnovación) y crecimiento económico. Posteriormente, la sección tres muestra lo que se ha venido haciendo en la Unión Europea en relación a la innovación y ecoinnovación, mientras que la siguiente sección es la metodología. La sección cinco contiene los resultados, y finalmente vienen las conclusiones. 


\section{Aspectos teóricos: innovación, ecoinnovación y crecimiento económico}

\subsection{Contribuciones teóricas a la relación de innovación y ecoinnovación sobre el crecimiento económico}

La relación entre innovación y crecimiento económico ha sido estudiada desde hace ya varias décadas; sin embargo, no necesariamente se hacía uso del término de innovación, sino que inicialmente se consideraba al progreso tecnológico. Schumepter fue el primero en considerar a la innovación como situación en donde un descubrimiento científico entra en el mercado y desplaza a productos similares (Aghion y Howitt, 1990). El término ocupado por Schumpeter es «destrucción creativa» y se relaciona con la posibilidad de que una empresa introduzca un nuevo producto que sustituya a uno ya existente y que disfrute por un periodo de tiempo (duración de la patente), de los beneficios de un monopolio.

Solow (1957) elaboró un modelo que explica el crecimiento económico de los países en función del capital, el trabajo y el progreso tecnológico, en donde esta última variable se incluye de forma exógena. Sala-i-Martín (2000) considera que el crecimiento económico de largo plazo está en función de la acumulación que se da del progreso tecnológico.

La corriente del crecimiento endógeno señala que el modelo clásico de Solow considera al progreso tecnológico como exógeno lo que hace que dicho modelo no logre explicar correctamente el crecimiento económico. Dicha corriente, considera al progreso tecnológico como endógeno, introducen en la función de producción además del capital y el trabajo, al capital humano, y consideraron que la generación de ideas o el progreso tecnológico incrementan la productividad, y se genera crecimiento económico (Romer, 1990; Grossman y Helpman, 1991).

La innovación como elemento central del crecimiento económico depende de múltiples factores como el stock de capital humano, el buen funcionamiento de los sistemas nacionales de innovación, de instituciones que defiendan los derechos de propiedad y de la cooperación que se dé entre investigadores (Lucas, 1988; Lundvall, 1992; North, 1989; Acemoglu, Johnson y Robinson, 2005; Rothaermel y Hess, 2007).

En general, la innovación genera un aumento de la productividad y efectos derrame en toda la economía, lo que contribuye al crecimiento económico. El vínculo entre innovación y crecimiento económico de largo plazo es fuerte (Aghion y Griffith, 2008)), y explica el crecimiento económico alto que han tenido países en las últimas décadas.

La ecoinnovación implica una «destrucción creativa» al estilo schumpeteriano, pero aplicada a una mejora en el medio ambiente de las actividades humanas. Kemp y Pearson (2007) consideran que la ecoinnovación, en el sentido del Manual de Oslo, como una mejora no solo de productos, sino que de modelo de negocios, una nueva forma de administración, etcétera, que tiene impacto en la firma, pero además que se reducen los impactos en el medio ambiente en sentido amplio, es decir, una reduc- 
ción de la contaminación y del uso de los recursos naturales. Tomado la definición de Kemp y Pearson, la ecoinnovación debe tener dos características: debe producir una «destrucción creativa» y al mismo tiempo debe hacer uso de una menor cantidad de recursos naturales y/o un menor impacto en ellos.

En varios foros internacionales se ha señalado la importancia del crecimiento verde, entendido este como aquel crecimiento de la economía que no tiene afectaciones significativas sobre el medio ambiente. En el año 2009 los países miembros de la OCDE adoptaron la estrategia para la crecimiento verde (OCDE, 2009), que tiene como objetivo alcanzar un desarrollo sustentable. Uno de los medios para alcanzar el crecimiento verde es el desacoplamiento del crecimiento económico con respecto al deterioro ambiental. Machiba (2010) establece que el desacoplamiento puede ser relativo o absoluto, en donde el primer término se refiere a que el crecimiento económico se desvincule del deterioro ambiental, a través de una reducción del uso de recursos por unidad de valor añadido, mientras que el segundo término se refiere a la reducción de la energía a niveles que sean sustentables para la economía.

La ecoinnovación puede contribuir a la economía verde, a través de productos, procesos y modelos negocios, etc. que contribuyan a la «destrucción creativa» schumpeteriana, pero que al mismo tiempo puedan lograr un menor impacto en el medio ambiente. La ecoinnovación puede ayudar que se dé un desacoplamiento del crecimiento económico y la degradación ambiental en términos relativos en el corto y mediano plazo, y absolutos en el largo plazo.

Saucedo (2013) muestra que las tecnologías ambientales pueden contribuir a un continuo crecimiento económico y al mismo tiempo una mejora considerable del medio ambiente. El autor señala que en el caso de la Unión Europea, las tecnologías ambientales pueden contribuir a que se alcance un desarrollo sustentable en el mediano y largo plazo.

\subsection{Estudios empíricos sobre los efectos de la innovación y ecoinnovación sobre el crecimiento económico}

Existen una gran cantidad de estudios empíricos sobre los determinantes del crecimiento económico, en esta sección nos enfocaremos solo a algunos que vinculan el crecimiento económico con la innovación.

Higino (2005) realiza un estudio con datos panel para analizar la relación entre crecimiento económico y una serie de variables, entre las cuales se encuentra la innovación, para países en desarrollo y desarrollados. El autor encuentra que con el método de efectos fijos hay una relación positiva entre crecimiento económico e innovación, sin embargo dicha relación no se mantiene para los países en desarrollo. Ulku (2004) mediante un modelo de datos panel y una metodología de efectos fijos encuentra que la innovación tiene un impacto positivo en el crecimiento económico de los países de la OCDE y de 10 no miembros de dicha organización. 
Hasan y Tucci (2010) estudian la relación innovación tecnológica y crecimiento económico para 58 países con un panel dinámico para el periodo 1980-2003. Los autores encuentran que hay una relación positiva entre las innovaciones tecnológicas y el crecimiento económicos de dicho grupo de países. Wong, Ho y Autio (2005) encuentran una relación positiva entre crecimiento económico e innovación tecnológica en un conjunto de 32 países. Los autores realizan una regresión para el año 2002 con una metodología de sección cruzada.

Demirel y Kesidou (2011) estiman un modelo Tobit a nivel de firma en Reino Unido, para analizar cómo afectan las políticas públicas externas a la empresa y factores internos de la empresa a los diferentes tipos de ecoinnovación. Los autores consideran 3 tipos de ecoinnovación: tecnologías de control de contaminación, tecnologías integradas de producción más limpia e investigación y desarrollo ambiental. Los resultados muestran que las tecnologías de control de la contaminación y las tecnologías integradas de producción más limpias son determinadas por decisiones de actualización del equipo para mejorar la eficiencia de la empresa, mientras que la inversión en investigación y desarrollo ambiental están determinadas por las regulaciones ambientales. Los factores internos de la empresa afectan a los dos primeros tipos de ecoinnovación, mientras que las políticas externas a la empresa afectan al tercer tipo de innovación.

Kesidou y Demirel (2012) aplican un modelo de selección Heckman a nivel de empresa en Reino Unido para determinar los factores que determina la ecoinnovación. Los autores consideran que hay tres determinantes teóricos de la ecoinnovación: las regulaciones ambientales, los factores de oferta como las capacidades de las empresas y factores de la demanda como los requerimientos de los consumidores sobre responsabilidad social corporativa. Los resultados muestran que los factores de la demanda afectan la decisión de las empresas de iniciar las ecoinnovaciones, sin embargo mayores inversiones en ecoinnovación son determinadas por factores internos de las empresas (ahorro de costos) y por los requerimientos de las regulaciones ambientales.

\section{Innovación y ecoinnovación en el contexto de la Unión Europea}

\subsection{Innovación en la Unión Europea}

Uno de los primeros programas europeos que apoya actividades relacionadas con la innovación, cómo es la investigación, inició en noviembre de 1971, el programa de Cooperación Europea en investigación Científica y Técnica (COST), el primero de tres pilares de iniciativas de investigación europea conjuntas. El programa es un marco intergubernamental que permite la coordinación de la investigación financiada a nivel nacional y europeo. Posteriormente, en 1984 se creó el primer programa marco (1984-1987) de Investigación y Desarrollo Tecnológico (RTD). Un año después la iniciativa intergubernamental de apoyo a la I+D a nivel europeo, EUREKA 
(European-wide Network for Industrial R\&D) fue creada para financiar proyectos europeos conjuntos. A diferencia del programa COST que se enfoca más hacia áreas de interés público, EUREKA provee financiamiento a proyectos que han sido concebidos, desarrollados y ejecutados por la industria privada. Es precisamente en los años noventa cuando se incluyen, explícitamente, políticas de innovación en documentos públicos.

La Comisión Europea publicó el «Libro Verde de la Innovación», que identifica los retos de la innovación en la UE y formula propuestas de acción elementos para vencer los retos y ampliar la capacidad de innovación en la región. Entre los objetivos más importantes que se persiguen se encuentran los siguientes: reforzar los esfuerzos de investigación directa hacia la innovación y los recursos humanos para la innovación, así como mejorar las condiciones de financiamiento para la innovación, entre otros. En el Libro Verde de la Innovación se considera a la innovación como «sinónimo de producir, asimilar y explotar con éxito una novedad, en las esferas económica y social, de forma que aporte soluciones inéditas a los problemas y permita así responder a las necesidad de las personas y de la sociedad» (Comisión Europea, 1995).

El primer plan de acción para la innovación en Europa que se lanzó en 1996 incluyó tres ámbitos de acción: promover una cultura de innovación, establecer un marco favorable y articular mejor la investigación e innovación. Este plan de acción reagrupó un número limitado de acciones a escala comunitaria que fueron definidas en el Libro Verde como esenciales en el proceso de innovación.

La Estrategia de Lisboa marcó un hito en la política de innovación de la Unión Europea en el 2000. El Consejo Europeo lanzó un objetivo estratégico que consistía en convertir a la economía de la UE en la más competitiva del mundo para el 2010. Sin embargo, la mayoría de los expertos coinciden en que, en general, la Estrategia de Lisboa fracasó. Varios factores externos influyeron como los ataques terroristas a los Estados Unidos de América el 11 de septiembre de 2001 y la explosión de la «burbuja puntocom» en el 2000. Una revisión de la estrategia encabezada por Wim Kok (2004) concluyó que aún y cuando se logró un avance, la mayoría de los objetivos no se alcanzaron. Si bien la estrategia ayudó a crear un consenso amplio acerca de las reformas necesarias para la UE, la implementación fue difícil por las estructuras débiles de gobernanza y falta de comunicación sobre los beneficios de la estrategia y las implicaciones que tendría no llevar a cabo las reformas necesarias.

Europa 2020 es la estrategia de la UE para el crecimiento y el empleo (20102020). La UE busca superar la crisis, paliar las diferencias de su modelo de crecimiento y crear las condiciones necesarias para un crecimiento inteligente, sostenible e integrador. La UE elaboró una nueva política de desarrollo e innovación, iniciativa emblemática Unión por la Innovación, en el marco de la Estrategia Europa 2020 para apoyar la innovación en ámbitos como el cambio climático, eficiencia energética, seguridad alimentaria y salud, entre otros.

Horizonte 2020, es un programa de financiación de I+D de la UE dotado de un presupuesto inicial de 71.000 millones de euros para el periodo 2014-2020. Entre 
los objetivos de este programa se encuentran el crear una ciencia de excelencia que refuerce la posición de la UE a nivel mundial y hacer de Europa un lugar más atractivo para invertir en investigación e innovación. Es el instrumento financiero para implementar la Unión por la Innovación y la iniciativa Europa 2020 para asegurar la competitividad europea.

En diciembre de 2013, el Consejo Europeo aprobó las normas que regirán la inversión de la política de cohesión de la UE para el periodo 2014-2020. La estrategia de especialización inteligente en investigación e innovación (RIS3) de la Comisión Europea tiene como objetivo que los Estados miembros y regiones de la UE identifiquen las especializaciones de conocimiento que mejor se ajusten con su potencial de innovación considerando sus recursos y capacidades.

\subsection{Ecoinnovación en el contexto europeo}

Las llamadas «industrias verdes» forman parte de las industrias de la UE que, a pesar de la crisis financiera de 2008, han crecido en ingresos y puestos de trabajo. La ecoinnovación para la UE tiene un potencial no solo como generadora de empleo sino también del crecimiento. La política medioambiental europea está realizando esfuerzos para integrar la ecoinnovación en las políticas medioambientales e industriales.

El VII Programa de Acción en materia de Medio Ambiente (7EAP) vigente hasta el 2020 se articula en torno a la visión de «vivir bien, respetando los límites de nuestro planeta». Este programa debe contribuir a alcanzar los objetivos medioambientales y de cambio climático de la UE.

Existen varias definiciones de la ecoinnovación en la literatura, pero no hay consenso al respecto. En este artículo de investigación presentaremos una definición que consideramos importante.

En el Plan de Acción de la Ecoinnovación (2011) (European Commission, 2011) la ecoinnovación es:

«cualquier innovación cuyos objetivos se orienten al desarrollo sostenible a través del impacto sobre el medio ambiente, el aumento de la resiliencia frente a las presiones medioambientales o un uso más eficiente y responsable de los recursos naturales».

Podemos decir que la diferencia entre una innovación y las ecoinnovaciones es que en las ecoinnovaciones además de un beneficio económico también hay un beneficio medioambiental. Los resultados de la ecoinnovación pueden ser, por ejemplo, mayor productividad de los recursos y/o menor emisiones de gases de efecto invernadero, entre otros.

Mientras que algunos países europeos consideran que la investigación y la innovación como claves para salir de la crisis y han podido mantener sus actividades 
públicas de I+D, otros no han podido mantener sus niveles de inversión para sus políticas de investigación e innovación ni tampoco retener su talento (fuga de cerebros) y se enfrentan a obstáculos importantes para impulsar la innovación.

A nivel europeo, la ecoinnovación se está convirtiendo en una estrategia clave de la competitividad que puede aplicarse en todos los sectores de actividad y en todo tipo de organizaciones.

\section{Metodología}

\subsection{Descripción y desarrollo del modelo}

En esta sección se analiza la relación existente entre crecimiento económico, ecoinnovación y una serie de variables económicas. Para la contrastación empírica del modelo sobre la ecoinnovación se utilizó la técnica de panel de datos en la que se analiza la relación entre crecimiento económico y ecoinnovación. A continuación se presenta una descripción de la base de datos, después se presenta la descripción del modelo de panel y su estimación.

\subsection{Datos}

Los datos provienen del Observatorio sobre EcoInnovación (EIO), el Banco Mundial (BM) y la Oficina Europea de Estadísticas (EUROSTAT). La unidad de análisis es el país para el periodo 2002-2010. Los datos de ecoinnovación del EIO solo están disponibles para el periodo 2010-2013 para los 28 países de la UE. Se utilizaron solo tres variables (del total de las que integran el índice ecoinnovación) que tenían una temporalidad mayor, con el objetivo de tener un panel con un mayor número de años, sin embargo para la construcción del panel no estaban disponibles dichas variables para todos los países de la UE, por lo que finalmente se tuvieron datos para 11 países de la UE y tres variables de ecoinnovación en el periodo 2002-2010 (Cuadro 1).

Las variables de ecoinnovación se complementaron con la variable dependiente, PIB per cápita, y otras variables independientes provenientes del BM y EUROSTAT (Cuadro 2). 


\section{CUADRO 1}

VARIABLES DISPONIBLES DEL ÍNDICE DE ECOINNOVACIÓN

\begin{tabular}{|c|l|l|l|}
\hline Periodo & \multicolumn{1}{|c|}{ Países } & \multicolumn{1}{c|}{ Bloque } & \multicolumn{1}{c|}{ Variables } \\
\hline & $\begin{array}{l}\text { Bulgaria, Finlandia, } \\
\text { Hungría, Italia, Letonia, } \\
\text { Polonia, Portugal, } \\
\text { Rumania, Eslovaquia, } \\
\text { Eslovenia, España }\end{array} 2002-$ & $\begin{array}{l}\text { 1. Entradas de } \\
\text { ecoinnovación }\end{array}$ & $\begin{array}{l}\text { 1.2. Total de personal e } \\
\text { investigadores en I+D } \\
\text { (porcentaje del empleo } \\
\text { total). }\end{array}$ \\
\cline { 3 - 4 } & & $\begin{array}{l}\text { 2. Actividades de } \\
\text { ecoinnovación }\end{array}$ & $\begin{array}{l}\text { 2.3. Organizaciones } \\
\text { registradas con ISO 14001 } \\
\text { (por millón de habitantes). }\end{array}$ \\
\cline { 3 - 4 } & & $\begin{array}{l}\text { 5. Resultados } \\
\text { socioeconómicos }\end{array}$ & $\begin{array}{l}\text { 5.1. Exportación de } \\
\text { productos de ecoindustrias } \\
\text { (porcentaje de las } \\
\text { exportaciones totales). }\end{array}$ \\
\hline
\end{tabular}

FUENTE: Elaboración propia con información del Observatorio de Ecoinnovación.

\section{MODELO DE ECOINNOVACIÓN Y CRECIMIENTO ECONÓMICO}

\begin{tabular}{|c|l|l|}
\hline $\begin{array}{c}\text { Variable } \\
\text { dependiente }\end{array}$ & \multicolumn{1}{|c|}{ Variables independientes } & \multicolumn{1}{|c|}{ Identificador } \\
\hline \multirow{4}{*}{$\begin{array}{c}\text { PIB per cápita } \\
\text { (PIBPER) }\end{array}$} & Total de inversión en el medio ambiente por la industria. & TINVIND \\
\cline { 2 - 3 } & Total de inversión en el medio ambiente por el gobierno. & TINGOB \\
\cline { 2 - 3 } & Total de gasto corriente en medio ambiente por la industria. & TGASIND \\
\cline { 2 - 3 } & Total de gasto corriente en medio ambiente por el gobierno. & TGASGOB \\
\cline { 2 - 3 } & Inscripción escolar nivel terciario. & AHBRU \\
\cline { 2 - 3 } & Número de certificados ISO 14001. & INESTER \\
\cline { 2 - 3 } & Total de personal en I+D. & ISO \\
\cline { 2 - 3 } & Población Activa Total. & TOTINV \\
\cline { 2 - 3 } & Ingresos fiscales ambientales. & POBACT \\
\hline & Exportación de productos de ecoindustrias. & INFISAM \\
\hline
\end{tabular}

FUENTE: elaboración propia con información del Observatorio de Ecoinnovación, el Banco Mundial y la Oficina Europea de Estadísticas. 


\subsection{Descripción de las variables}

\subsubsection{Variable dependiente}

Crecimiento económico: cambio anual del PIB per cápita, el BM la define como el producto interno bruto dividido por la población a mitad de año, los datos se expresan en dólares de los Estados Unidos a precios constantes del 2005.

\subsubsection{Variables dependientes}

Total de inversión en el medio ambiente: EUROSTAT la define como la inversión en protección del medio ambiente incluye todas las erogaciones determinadas en un año para la maquinaria, el equipo y la tierra. Las inversiones totales en un sector o industria es la suma de dos categorías: 1) inversiones end-of-pipe (tratamiento de la contaminación), y 2) las inversiones en tecnologías integradas (inversiones en prevención de la contaminación).

Total de gasto corriente en medio ambiente: el gasto corriente total para la protección del medio ambiente incluye tanto el gasto corriente interno y tasas/compras. El gasto corriente interno incluye el uso de energía, materiales, mantenimiento y personal propio para medidas realizadas por un sector para proteger el medio ambiente. Las tasas/compras incluyen todas las compras individuales de los servicios de protección del medio ambiente, desde productores privados y públicos. Estos pagos están vinculados con una actividad de protección del medio ambiente efectuada fuera de la empresa.

Ahorro bruto: el BM calcula el ahorro bruto como el ingreso nacional bruto menos el consumo total más las transferencias netas. El indicador fue extraído con cifras estimadas como porcentaje del PIB.

Inscripción escolar nivel terciario: es definida por el BM como la tasa bruta total de matrícula en educación superior. Corresponde al número total de estudiantes matriculados en educación superior (niveles 5 y 6 de la Clasificación Internacional Normalizada de la Educación), independiente de su edad, expresado como porcentaje de la población total del grupo etario cinco años después de finalizar la enseñanza secundaria.

Número de certificados ISO 14001: este indicador diseñado por el EIO proporciona una visión general de la norma ISO 14,001 de empresas certificadas en los países europeos. La norma ISO 14.001 es una norma internacional que ayuda a una organización a identificar, priorizar y gestionar los riesgos ambientales. Es parte de la familia ISO 14000, que ayuda a las empresas a desarrollar su propio sistema de gestión ambiental. 
Total de personal en I+D: Corresponde al total de personal e investigadores en I+D como porcentaje de la fuerza laboral total y empleo total.

Población Activa Total: Comprende a personas de 15 años o más que aportan trabajo para la producción de bienes y servicios durante un periodo específico. Incluye tanto a las personas con empleo como a las personas desempleadas. La población activa incluye a las fuerzas armadas, a los desempleados, los que buscan su primer trabajo, y excluye a quienes se dedican al cuidado del hogar y a otros trabajadores y cuidadores no remunerados, de acuerdo con el BM.

Ingresos fiscales ambientales: El reglamento número 691/2011 de la UE define un impuesto ambiental a aquel impuesto cuya base fiscal es una unidad física (o su equivalente de unidad física) de algo que tiene un probado impacto específico negativo sobre el medio ambiente, el cual es identificado en el Sistema Europeo de Cuentas Nacionales y Regionales como un impuesto.

Exportación de productos de ecoindustrias: El EIO lo define como la participación de las exportaciones de ecoindustrias en las exportaciones totales (en porcentaje). Basados en 25 códigos comerciales utilizados para la exportación a partir del análisis Ecotec. Los subsectores analizados son: el control de la contaminación del aire, el control de la contaminación del agua, eliminación de residuos, equipos de vigilancia, otros equipos del medio ambiente, energía solar térmica, fotovoltaica y energía hidroeléctrica.

El Cuadro 3 resume el total de variables utilizadas en ambos modelos, así como los detalles específicos de cada una. 


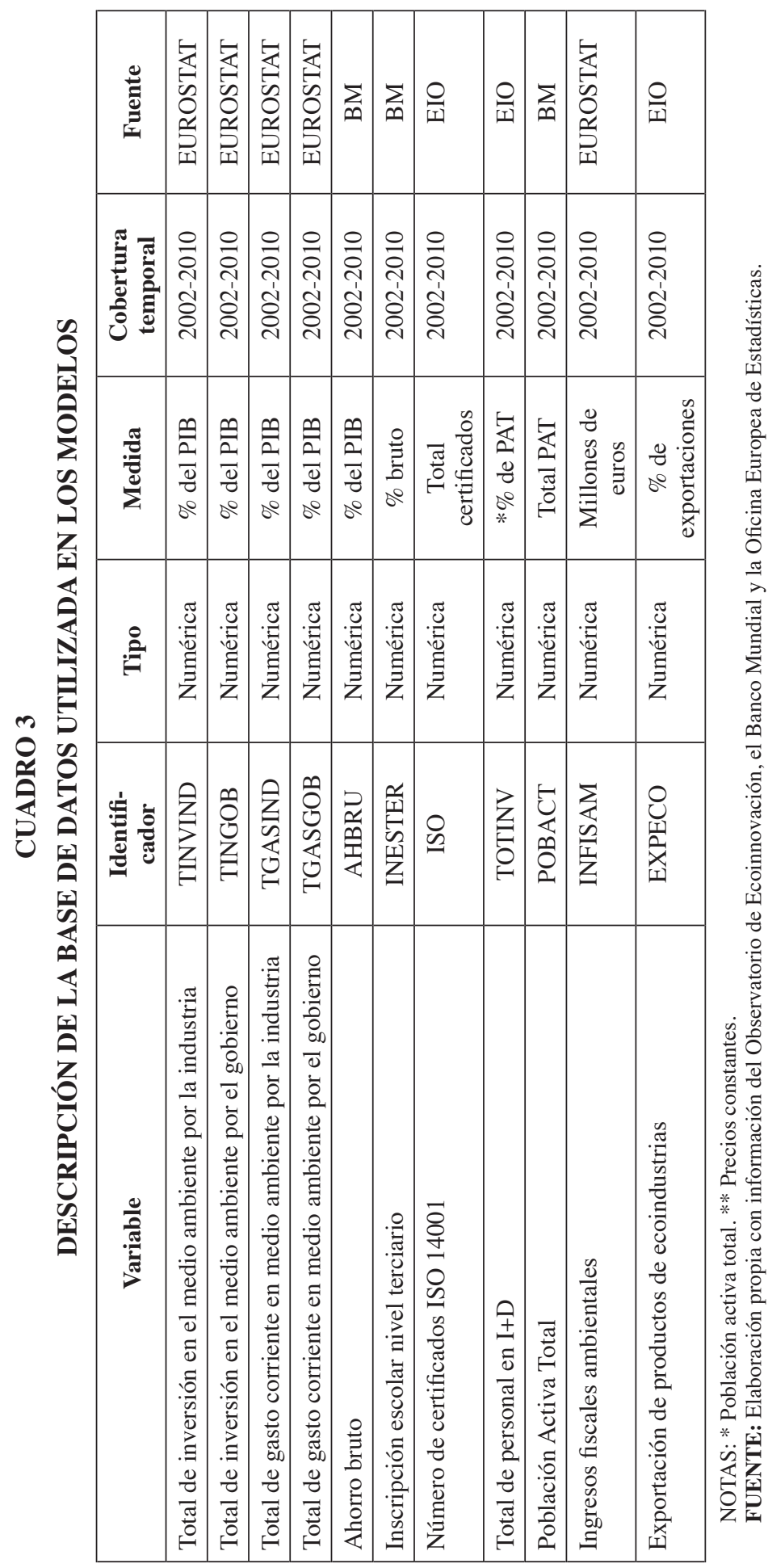




\subsection{Metodología econométrica}

El modelo utilizado es de datos panel, y tiene ventajas con respecto a las formas tradicionales de análisis de datos. La técnica de datos en panel incluye información transversal y temporal al mismo tiempo.

Un modelo de regresión con datos de panel tiene la siguiente especificación:

$$
y_{i t}=\mu_{i t} \beta_{i t}+u_{i t} \text { para } i=1,2, \ldots, N ; \quad t=1,2, \ldots, T
$$

Para estimar parámetros con datos panel se pueden utilizar modelos de efectos fijos y aleatorios, y dentro de los modelos de efectos fijos hay varios métodos. El modelo de efectos fijos tiene como desventaja que hay demasiados parámetros por estimar y una gran pérdida de grados de libertad. Si los efectos se supongan fijos o aleatorios no representa una cualidad intrínseca de la especificación (Arellano y Bover, 1991).

Con el modelo de efectos aleatorios se supone que la heterogeneidad es aleatoria, además de que las variables observables, $x_{i t}$, no están correlacionadas con el término no observable $\mu_{\mathrm{i}}$ ni con el término estocástico.

La estimación para los parámetros se realizó por medio de mínimos cuadrados generalizados, y el modelo empleado para la estimación fue el de efectos aleatorios.

\section{Resultados}

El Cuadro 4 muestra las variables que inciden sobre la variable crecimiento económico son: el total de inversión en el medio ambiente por el gobierno, el total de gasto corriente en el medio ambiente por la industria, el ahorro bruto, la inscripción escolar nivel terciario, el número de certificados ISO 14001 de empresas europeas, el total de personal en I+D, el total de población activa, los ingresos fiscales ambientales y las exportaciones de productos de ecoindustrias.

La variable inversión total en el medio ambiente por el gobierno, incide positivamente sobre el crecimiento económico, debido a que es estadísticamente significativa. Por otro lado, el gasto total corriente en el medio ambiente por parte de la industria resultó ser significativo, sin embargo el valor es negativo, lo que indica que la inversión resulta ser un costo para la industria en el corto plazo.

El coeficiente de ahorro bruto resultó ser significativo y positivo, por lo que un incremento del ahorro produce un aumento del PIB per cápita.

Los países que tienen un mayor número de estudiantes matriculados en educación superior indicen de forma negativa sobre el crecimiento económico, esto posiblemente a que los gobiernos absorben una gran parte del gasto en educación.

Las empresas que se encuentran dentro de países europeos y que optan por la certificación ISO 14001 generan un efecto negativo sobre el crecimiento económico, debido a que un aumento en una unidad de certificados ISO 14001, cuando todos los 


\section{CUADRO 4}

RESULTADOS DEL MODELO DE EFECTOS ALEATORIOS:

CRECIMIENTO ECONÓMICO

\begin{tabular}{|c|c|c|}
\hline \multicolumn{3}{|c|}{ Variable dependiente: PIBPER } \\
\hline Variables independientes & Coeficiente & Valor $\mathbf{p}$ \\
\hline TINVIND & $\begin{array}{c}-347,76 \\
(2.998,01)\end{array}$ & 0,908 \\
\hline TINGOB & $\begin{array}{c}4.863,25 \\
(2.270,08)\end{array}$ & 0,032 \\
\hline TGASIND & $\begin{array}{c}-10.110,48 \\
(2.041,43)\end{array}$ & 0,000 \\
\hline TGASGOB & $\begin{array}{l}-3.265,00 \\
(2.594,94)\end{array}$ & 0,208 \\
\hline AHBRU & $\begin{array}{l}325,69 \\
(75,28)\end{array}$ & 0,000 \\
\hline INESTER & $\begin{array}{c}-171,06 \\
(32,62)\end{array}$ & 0,000 \\
\hline ISO & $\begin{array}{c}-0,25 \\
(0,097)\end{array}$ & 0,009 \\
\hline TOTINV & $\begin{array}{c}13.851,17 \\
(654,27)\end{array}$ & 0,000 \\
\hline POBACT & $\begin{array}{l}-0,00006 \\
(0,00001)\end{array}$ & 0,000 \\
\hline INFISAM & $\begin{array}{c}0,5842 \\
(0,0534)\end{array}$ & 0,000 \\
\hline EXPECO & $\begin{array}{c}3.012,64 \\
(1.083,35)\end{array}$ & 0,005 \\
\hline Constante & $\begin{array}{c}4.979,36 \\
(2.180,78)\end{array}$ & 0,022 \\
\hline sigma u & 0 & \\
\hline sigma_e & 771,98174 & \\
\hline Rho & 0 & \\
\hline \multicolumn{3}{|l|}{$\mathrm{R}$} \\
\hline Intra grupos & 0,0386 & \\
\hline Entre grupos & 0,9886 & \\
\hline General & 0,9600 & \\
\hline
\end{tabular}

NOTA: El error estándar se presenta entre paréntesis debajo de cada coeficiente. 12.0 .

FUENTE: Elaboración propia con información de EUROSTAT, BM, EIO, a través del software Stata 
demás factores permanecen constantes, proporciona una disminución del PIB per cápita. Esto posiblemente a que la empresa tiene que reestructurarse para cumplir con la certificación lo que puede llevarla a incurrir en gastos adicionales.

El personal que se dedica a la investigación y el desarrollo genera un efecto positivo sobre el crecimiento económico. Podríamos suponer que si bien a los países les cuesta invertir en educación superior, a la larga está generando una mayor cantidad de investigadores, los cuales están contribuyendo al crecimiento económico.

La población activa a pesar de haber resultado significativa, tuvo efectos casi nulos sobre el crecimiento económico, debido a que su coeficiente es negativo y muy cercano a cero.

Un aumento en los ingresos fiscales ambientales como producto de un impuesto ambiental incide positivamente sobre el crecimiento económico para los países europeos. Si los ingresos fiscales ambientales aumentan una unidad, permaneciendo los demás factores constantes, generará un incremento marginal en el PIB per cápita, las respuestas a una incidencia pequeña como la observada sobre el crecimiento económico pueden ser muchas, entre ellas puede deberse a que las empresas se están reorientando al grupo de empresas «verdes» como parte del fomento de la estrategia europea y, por tanto, no se vean tan afectadas por un impuesto ambiental, otra posibilidad es que los impuestos ambientales no sean parte de las medidas optadas por los países europeos como parte de su estrategia de crecimiento sostenible, por lo que los ingresos fiscales ambientales son relativamente bajos.

La exportación de productos de las ecoindustrias tienen un impacto positivo sobre el crecimiento económico, debido a que su coeficiente resultó positivo y significativo. Esto podría ser prueba de que las tecnologías verdes están generando la apertura de nuevos mercados con posibilidades de crecimiento económico como parte de su ventaja competitiva.

\section{Conclusiones}

Desde el punto de vista teórico la innovación contribuye al crecimiento económico, debido a que permiten que las nuevas ideas se masifiquen, lo que contribuye a que se reduzcan costos económicos. Por otro lado, la ecoinnovación es herramienta muy importante para reducir el impacto de las actividades económicas sobre el medio ambiente, sin que al hacerlo se incurre en un alto costo económico.

Las instituciones de la Unión Europea han venido impulsado políticas públicas que favorezcan el cuidado del medio ambiente, y concretamente en el 2011 se lanzó el Plan de Acción para la Ecoinnovación que buscan un menor impacto de las actividades económicas sobre el medio ambiente a través de las innovaciones ambientales.

El objetivo planteado del texto fue analizar empíricamente si la ecoinnovación tiene una relación positiva sobre el crecimiento económico de 11 países de la Unión Europea. Los resultados muestran que la ecoinnovación ha contribuido positivamente al crecimiento económico en el periodo 2002-2010 en los 11 países de la Unión Europea. 
Los resultados econométricos muestran que la inversión en el medio ambiente de los gobiernos, los ingresos fiscales ambientales y la exportación de productos de ecoindustrias tienen un impacto positivo sobre el crecimiento económico.

Los resultados del texto muestran que las políticas públicas en favor del medio ambiente no tienen un costo excesivamente alto en términos del PIB, por lo que dichas políticas podrían ser utilizadas por los gobiernos para alcanzar objetivos ambientales sin incurrir en un excesivo costo económico.

No se incluyeron a todos los miembros de la Unión Europea, debido a la falta de series unificadas para todos los países, por lo que los resultados deben ser tratados con cautela, sin embargo son una primera aproximación para el caso de los países europeos, por lo que consideramos que representan una aportación original para la discusión de la política ambiental europea y los costos económicos de la misma.

\section{Referencias bibliográficas}

[1] ACEMOGLU, D.; JOHNSON, S. y ROBINSON, J. A. (2005). «Institutions as a fundamental cause of long-run growth». Handbook of Economic Growth, 1, 385-472.

[2] AGHION, P. y GRIFFITH, R. (2008). Competition and Growth: Reconciling Theory and Evidence. Cambridge, The MIT Press.

[3] AGHION, P. y HOWITT, P. (1990). «A model of growth through creative destruction». National Bureau of Economic Research No. w3223.

[4] ARELLANO, M. y BOVER, O. (1991). «La econometría de datos en panel». Investigaciones Económicas, XIV (1), 3-45.

[5] DEMIREL, P. y KESIDOU, E. (2011). «Stimulating different types of ecoinnovation in the UK: Government policies and firm motivations». Ecological Economics, 70 (8), 1546-1557.

[6] EUROPEAN COMMISSION (1995). Common Actions for Growth and Employment: The Community Lisbon Programme, Brussels.

[7] EUROPEAN COMMISSION (2011a). State of the Innovation Union 2011, Brussels.

[8] EUROPEAN COMMISSION (2011b). Innovation for sustainable Future - The Ecoinnovation Action Plan (EcoAP), Brussels.

[9] GROSSMAN, G. y HELPMAN, E. (1991). Innovation and Growth in the Global Economy. Cambridge, The MIT Press.

[10] HASAN, I. y TUCCI, C. (2010). «The innovation-economic growth nexus: Global evidence». Research Policy, 39 (10), 1264-1276.

[11] KEMP, R. y PEARSON, P. (2007). Final report MEI project about measuring ecoinnovation. Maastricht, UM MERIT.

[12] KESIDOU, E. y DEMIREL, P. (2012). «On the drivers of ecoinnovations: Empirical evidence from the UK». Research Policy, 41 (5), 862-870.

[13] KOK, W. (2004). Facing the Challenge: The Lisbon strategy for growth and employment. Luxembourg, Brusels.

[14] LUCAS, R. E. (1988). «On the Mechanics of Economic Development». Journal of Monetary Economics, 22 (1), 3-42.

[15] LUNDVALL, B. (1992). National Systems of Innovations. Londres, Pinter. 
[16] MACHIBA, T. (2010). «Ecoinnovation for enabling resource efficiency and green growth: development of an analytical framework and preliminary analysis of industry and policy practices». International Economics and Economic Policy, 7 (2-3), 357-370.

[17] NORTH, D. C. (1989). «Institutions and economic growth: An historical introduction». World Development, 17 (9), 1319-1332.

[18] OECD (2009). «Declaration on green growth, adopted at the council meeting at ministerial level». OCDE, Paris. Recuperado en febrero de 2016 de www.oecd.org/document/ 63/0,3343,en_2649_201185_43164671_1_1_1_1,00.html

[19] ROMER, P. (1990). «Endogenous Technological Change». Journal of Political Economy, 98 (5), S71-S102.

[20] ROTHAERMEL, F. T. y HESS, A. M. (2007). «Building dynamic capabilities: Innovation driven by individual, firm, and network-level effects». Organization Science, 18 (6), pp. 898-921.

[21] SALA-I-MARTIN, X. (2000). Apuntes de crecimiento económico. Barcelona, Antoni Bosch.

[22] SAUCEDO, E. (2013). «The European Union promoting the ecoinnovation: The environmental Technologies action plan», en G. Carrillo (coord.), Sistemas de gestión y modelos de organización para la innovación. México, Plaza y Valdez.

[23] SCHNEIDER, P. H. (2005). «International trade, economic growth and intellectual property rights: A panel data study of developed and developing countries». Journal of Development Economics, 78 (2), 529-547.

[24] SOLOW, R. (1957). «Technical change and the aggregate production function». The Review of Economics and Statistics, 39 (3), 312-320.

[25] ULKU, H. (2004). «R\&D, Innovation, and Economic Growth: An Empirical Analysis». IMF Working Papers, 04.

[26] WONG, P. K.; HO, Y. P. y AUTIO, E. (2005). «Entrepreneurship, Innovation and Economic Growth: Evidence from GEM data». Small Business Economics, 24 (3), 335-350. 